\title{
Antiangiogenesis Therapy
}

National Cancer Institute

\section{Source}

National Cancer Institute. Antiangiogenesis Therapy. NCI Thesaurus. Code C15568.

A therapeutic regimen that uses synthetic compounds or natural substances to prevent the development of new blood vessels. 